\title{
Fluorescent quantitation method for differentiating the nativity of green fluorescent protein
}

\begin{abstract}
A gel imaging method was employed to quantitate the GFP that had been subjected to denaturation and degradation treatments. This method is able to differentiate the nativity of GFP by relating the observed changes in the position of fluorescent bands which is unable to be detected using the spectrofluorometric method.
\end{abstract}

Keyword: Degradation; Denaturation; Fluorescence quantitation; Gel imaging method; Green fluorescent protein; Nativity; Spectrofluorometer 